\title{
Stability analysis of blood flow in multilayered viscoelastic tubes
}

\author{
N. N. KIZILOVA $\dagger$ and M. HAMADICHE $\$$
}

$\dagger$ Department of Theoretical Mechanics, Kharkov National University, Ukraine $\ddagger$ Laboratory of Mechanics of Fluids and Acoustics, Ecole Centrale de Lion, France

\section{Introduction}

Blood flow in arteries and veins as collapsible tubes is determined by fluid-structure interaction. Multilayered structure of the vessel wall and mechanical properties of its layers may influence the flow stability, flow rate and wall oscillations. Material parameters of the layers are estimated in experiments (Qilian 1993, Vito 2003).

\section{Materials and methods}

Stability of the flow of an incompressible Newtonian liquid in the multilayered tethered compliant tube is investigated. The wall of the tube is consisted of three layers with different mechanical properties. The governing equations are presented by Navier-Stokes equations for the liquid and momentum equations for the viscoelastic layers. Continuity conditions for velocity and stress fields at the fluid-solid and solid-solid interfaces are considered. The outer layer is attached to the surrounding tissues. Solution of the problem has been considered as a superposition of the Hagen-Poiseuille velocity field in the fluid and the displacement fields in the layers and small-amplitude axisymmetric disturbances in the form of normal mode. The first order system of differential equations for amplitudes of the disturbances has been obtained and investigated. The correspondent numerical method is presented in (Hamadiche and Gad-el-Hak 2004).

\section{Results and discussion}

Theoretical analysis and calculations have revealed multifarious influence of the shear module $\mathrm{G}^{1-3}$ and viscosities $\mu_{\mathrm{w}}^{\mathrm{j}}$ of the layers $j=1,2,3$ on stability of the flow. Some results are presented in figures 1 and 2. The isotropic uniform single-layered tube has one unstable

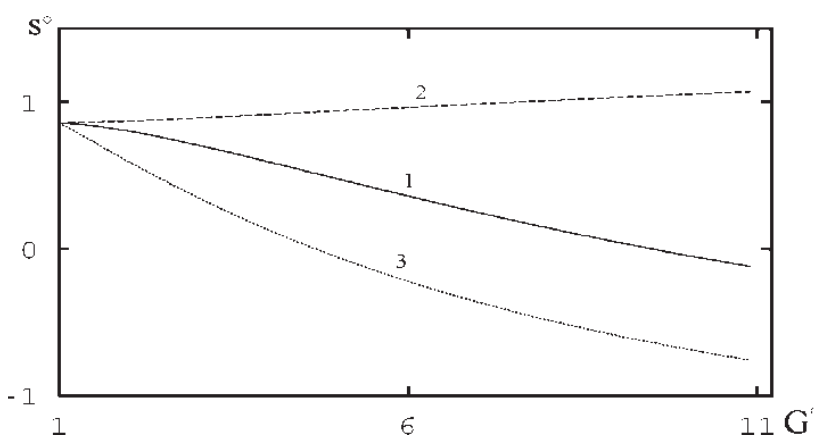

Figure 1. Amplification rate $s^{\circ}$ of the unstable mode versus the dimensionless shear modulus $G^{\circ}$ of one of the layers $j=1,2,3$ (curves $1-3$ ) while $\mathrm{G}_{\mathrm{k}}^{\circ}=1$ for $\mathrm{k} \neq \mathrm{j}$.

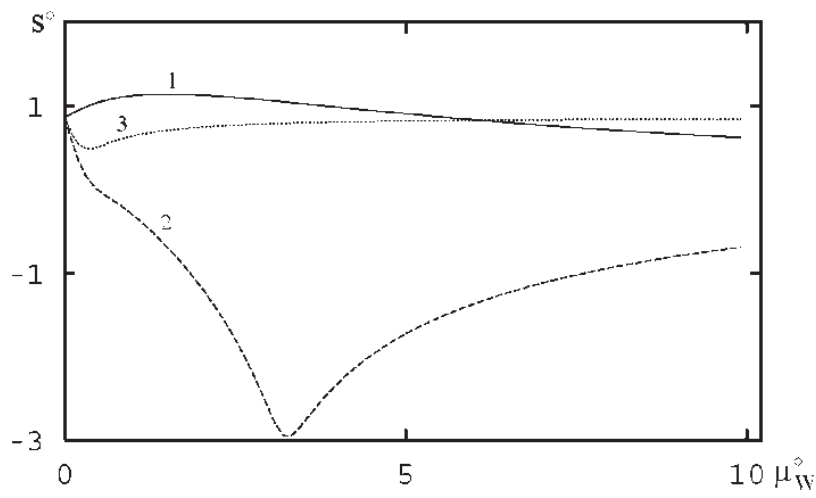

Figure 2. Amplification rate $s^{\circ}$ of the unstable mode versus the dimensionless viscosity $\mu_{\mathrm{w}}^{\circ}$ of one of the layers $\mathrm{j}=1,2,3$ (curves $1-3$ ) while $\mu_{\mathrm{wk}}^{\circ}=0$ for $\mathrm{k} \neq \mathrm{j}$.

mode. Increasing $\mathrm{G}^{1,3}$ and $\mathrm{G}^{2}$ exhibit a stabilizing and destabilizing effects on this mode accordingly. For high values $\mathrm{G}^{1,3}$ within the physiological range the system becomes stable. A small increase of $\mu_{\mathrm{w}}^{1}$ leads to increasing the amplification rate of the unstable mode whereas increasing $\mu_{\mathrm{w}}^{2}$ stabilizes the system. The comparative study of the uniform single-layered and the three-layered tubes revealed significant influence of the mechanical 
parameters and relative thicknesses of the layers on stability of the system. As far as thickening and increasing rigidity of separate layers are proper for different pathologies (hypertension, atherosclerosis etc.) the stability of the vessel can be changed by the variations of the material parameters and the flow rate can be essentially decreased in the case.

\section{Conclusions}

Multi-layered structure and mechanical parameters of the layers significantly influence flow stability in the compliant tubes. Variations of the elastic and viscous parameters of one of the layer or a few layers simultaneously exert a great influence on the stable and unstable modes. The results can be used for understanding the dynamics of the vessel wall as well as for construction and optimization of the multilayered coating for technical applications.

\section{References}

M. Hamadiche and M Gad-el-Hak, "Spatiotemporal stability of flow through collapsible, viscoelastic tubes", AIAA J., 122, pp. 772-786, 2004.

Y. Qilian, J. Zhou and Y.C. Fung, "Neutral axis location in bending and Young's modulus of different layers of arterial wall", Am. J. Physiol., 265, pp. H52-H60, 1993.

R.P. Vito and S.A. Dixon, "Blood vessel constitutive models 1995 2002”, Ann. Rev. Biomed. Eng., 5, pp. 413-439, 2003. 